\title{
Retraction
}

\section{Retracted: PASS-Predicted Hepatoprotective Activity of Caesalpinia sappan in Thioacetamide-Induced Liver Fibrosis in Rats}

\author{
The Scientific World Journal \\ Received 21 March 2018; Accepted 21 March 2018; Published 12 August 2018 \\ Copyright (C) 2018 The Scientific World Journal. This is an open access article distributed under the Creative Commons Attribution \\ License, which permits unrestricted use, distribution, and reproduction in any medium, provided the original work is properly \\ cited.
}

The Scientific World Journal has retracted the article titled "PASS-Predicted Hepatoprotective Activity of Caesalpinia sappan in Thioacetamide-Induced Liver Fibrosis in Rats" [1]. Figures 3(c) and 3(f) were reused from Figures 1(c) and 1(d) in an article by the same authors, Kadir et al. [2].

An institutional investigation by the University of Malaya found there was no system to index and file data and images to avoid mislabeling and mishandling, which led to errors and duplication of research data. The authors did not thoroughly check the manuscript before submission.

The authors said that both images (1(c) and $1(\mathrm{~d}))$ in Figure 1 in [1] are correct and the same as illustrated in the thesis by Kadir [3]. Panel (c) in both figures represented the same reference group (silymarin group-SY) because they ran two experiments in rats treated with different plants, but with the same reference group. This is also illustrated in Kadir's thesis [3]. Therefore, panel (c) is correct in both Figure $3(c)$ in [1] and Figure 1(c) in [2]. However, Figure 3(f) was mistakenly uploaded in this article, which probably happened due to incorrect labelling during data collection. This is also illustrated in the thesis [3].

The authors offered to provide replacement figures, but the Editorial Board recommended retraction.

\section{References}

[1] F. A. Kadir, N. M. Kassim, M. A. Abdulla, B. Kamalidehghan, F. Ahmadipour, and W. A. Yehye, "PASS-predicted hepatoprotective activity of Caesalpinia sappan in thioacetamide-induced liver fibrosis in rats," The Scientific World Journal, vol. 2014, Article ID 301879, 12 pages, 2014.

[2] F. A. Kadir, N. M. Kassim, M. A. Abdulla, and W. A. Yehye, "Hepatoprotective role of ethanolic extract of Vitex negundo in thioacetamide-induced liver fibrosis in male rats," EvidenceBased Complementary and Alternative Medicine, vol. 2013, Article ID 739850, 9 pages, 2013.

[3] F. A. Kadir, Hepatoprotective role of vitex negundo and caesalpinia sappan in thioacetamide-induced liver injury in rats [Ph.D. thesis], University Malaya, 2014. 